\title{
Intrapartal uterine avulsion with posterior cervical rupture
}

BY DUBRAVKO HABEK, TATJANA PAVELIĆ TURUDIĆ, IVAN ŠKLEBAR

\section{Abstract}

In this case study we describe an obstetric emergency of complete intrapartum left-lateral uterine avulsion, with posterior cervical rupture in a 31-year-old secundipara, following vacuum extraction. To the best of our knowledge, a similar case has not been previously reported in the literature. A live macrosomic male neonate was delivered by two tractions, with lateral episiotomy, and with shoulder dystocia that was relieved by McRoberts' and Resnik's maneuvers (Apgar score 7.8, birth weight/length 4640/57).

In our patient, the risk factors for avulsion and concomitant posterior cervical rupture included prolonged second stage of labor, delivering a macrosomic neonate in a secundipara with deflexion (parietal) in a cephalic presentation along the distended and thinned posterior uterine wall. Urgent total hysterectomy was performed in dramatical circumstances due to complete unilateral avulsion and cervical rupture, with continuous aortal compression, volume replacement and intact coagulation, which certainly contributed to the good final maternal outcome.

Key words: delivery, uterine avulsion, uterine rupture 


\section{Introduction}

Intrapartum complications, such as uterine rupture ( 1:1500 deliveries), are associated with a high perinatal mortality (neonatal 50\% and maternal 50\%) and morbidity. A higher rate of uterine rupture is recorded in women with previous injuries and operations such as cesarean section or myomectomy. According to localization, the anterior uterine segment is more frequently involved, while being quite atypical in the posterior uterine segment and non-scar uterus. (1-3)

Uterine avulsion (Fr. décollement) is a very rare and severe complication mostly presenting with signs of hemorrhagic shock and is generally associated with blunt abdominal or pelvic trauma and potential lethal outcome due to polytrauma. $(4,5)$ There are only a few literature reports of obstetric uterine avulsion (during pregnancy, at delivery and in the puerperium), mostly in association with blunt trauma, and very rarely occurring during or after delivery. The potential risk factors certainly include traumatic events, as well as aggressively applied fundal pressure and hyperpolysystolie. $(6,7)$

A case is described of complete left-sided intrapartum uterine avulsion with posterior cervical rupture in a secundipara following vacuum extraction. To the best of our knowledge, a similar report has not been previously reported.

\section{Case presentation}

A 31-year-old parturient woman was admitted to our maternity ward. Two years earlier, she had her first vaginal delivery in the $41^{\text {st }}$ week of gestation, associated with gestational diabetes, when she gave birth to a live macrosomic female, 4560/55, Apgar score 10/10. In her second pregnancy, the gestational diabetes was controlled by diabetic diet, which resulted in normal blood glucose levels. According to peripartum assessment, the child's weight was around $4500 \mathrm{~g}$. 
The course of the delivery was normal until the second stage of labor, which was prolonged. After $2 \mathrm{~h}$ in epidural analgesia, cardiotocography verified decelerations with labor pains 2-3/10, short, low-amplitude and inefficient with Syntocinon $囚$ (oxytocin) $5 \mathrm{IU} / 10 \mathrm{gtt} / \mathrm{min}$ stimulation; the cervix was fully open, and the head in the position with the largest diameter +1 in parietal presentation. Given the persistent head position, the prolonged second stage of labor and secondary uterine inertia in the secundipara, we decided to complete the delivery by vacuum extraction, for which the parents gave their consent. A live macrosomic male neonate was delivered at $2.32 \mathrm{p}$.m. by two tractions, with lateral episiotomy, and with shoulder dystocia that was relieved by McRoberts' and Resnik's maneuvers; the neonate was transferred to a neonatologist for further care (Apgar score 7.8, birth weight/length 4640/57). Fundal pressure was not applied. The placenta was delivered spontaneously following Syntocinon $®$ (oxytocin) 5 IU administration. The soft birth canal was revised and episiotomy sutured. In the fourth labor stage, at around 3.00 p.m., hypotension with tachycardia developed, while the uterus was contracted, no bleeding. Crystalloid and colloid volume replacement was ordered. Ultrasonography revealed some free fluid laterally along the uterus, more to the right. The abdomen was soft, no acute findings. A permanent urinary catheter was inserted. Blood pressure and pulse improved upon volume replacement, the patient was conscious, and hemogram and coagulogram laboratory findings were normal. At 4.55 p.m., blood pressure decreased again to 60/30, with tachycardia and presyncope; ultrasonography showed a greater amount of abdominal fluid without signs of acute abdomen, but with the abdomen sensitive to palpation laterally from the contracted uterus; therefore, urgent multi-slice computed tomography (MSCT) was ordered. Laboratory findings showed red blood cell count decline with intact coagulation, fibrinogen 3.5. Intensive resuscitation with crystalloids, colloids, tranexamic acid $2 \mathrm{~g}$ and blood products was initiated, while MSCT revealed massive intra-abdominal hemorrhage that required urgent exploratory laparotomy. The patient and her husband were informed in detail of the need for urgent, life-saving surgery, which they understood and gave their consent, including the 
possible procedural complications and hysterectomy, if necessary. Under general anesthesia, the abdomen was opened by lower median laparotomy to reveal an abundance of blood and coagulate. The uterus was contracted, completely avulsed from the left-lateral pelvic wall, with a denuded iliac fossa on the left and torn left adnexa and hematoma at the site of avulsion. A diffusely bleeding cervical transverse complex uterine rupture of 3-4 $\mathrm{cm}$ in length was found on inspection of the posterior wall (figure 1).

We decided to perform urgent hysterectomy due to impossible management of the avulsion and uterine rupture with appropriate hemostasis. Manual compression of the abdominal aorta was applied throughout the procedure. The vagina was sutured and urinary bladder intactness verified by methylene blue instillation, which showed normal findings. The abdomen was thoroughly explored, hemostasis and other intra-abdominal organ findings were normal. An abdominal drain was inserted and the abdominal cavity closed by layers. Throughout the procedure, the patient was hemodynamically stable, with high blood product replacement (9000 $\mathrm{mL}$ colloid and crystalloid, $510 \mathrm{~mL}$ fresh frozen plasma and $1550 \mathrm{~mL}$ erythrocyte concentrate). Urine was clear and diuresis normal.

The postoperative course was uneventful with thromboprophylaxis and early mobilization and antibiosis (amoxiclav and metronidazole). On postoperative day 9, the mother and the infant were discharged from the hospital in good general condition, with normal findings at one-month follow up visit.

\section{Discussion}

Complex intrapartum uterine lesions are rarely encountered. Obstetric avulsion has been reported in only a few cases in the early literature, in the form of complete manual avulsion of the uterus, uterine tube and ovaries, (8) and intrapartum avulsion of the round ligament. (9) Fu et al. recently described postpartum hemoperitoneum with hemorrhagic shock 
after normal vaginal delivery due to avulsion of the fibrous band between the right fallopian tube and the uterus, with active bleeding. (10) To the best of our knowledge, avulsion of the uterus from the pelvic wall has not yet been reported in the literature, in particular with a concomitant posterior uterine rupture; therefore, clinical discussion on the issue is certainly interesting but may be inadequate due to impossible comparison with literature data.

Uterine avulsion (ripping) from the surrounding structures during pregnancy and delivery can therefore be associated with altered topographic intra-abdominal relations, previous operative procedures (e.g., post-laparotomy adhesions), fibroids, or with excessive force acting upon the gravid uterus such as accidents, blunt injuries, or iatrogenic causes (e.g., excessive fundal pressure) (7) and other intrapartum lesions. In addition to these potential causes, Miller mentions rupture of non-scar uterus and vacuum extraction, oxytocin or prostaglandin induced delivery, multiparity, and fetal malpresentation, (3) which can also be considered as possible risk factors for uterine avulsion. Spontaneous obstetric or non-obstetric uterine avulsions have not yet been described. The isthmic-corporeal junction as a locus minoris resistentiae is the preponderant site of avulsion due to force action, whereas lateral avulsions are not known in clinical obstetrics. The authors generally opted for subtotal hysterectomy due to the complex nature of rupture and emergency with hemoperitoneum and hemorrhagic shock. (4-6) Preschany et al. (2) report a case of a 35-yearold pregnant woman with normal history and without any previous operative procedure. Following delivery with vacuum extraction, she presented on postpartum day 13 for weakness and severe abdominal pain. Magnetic resonance imaging showed a hematoma in the right parametria, therefore laparotomy was performed to reveal longitudinal uterine rupture from the isthmus to the fundus with hematoma. The patient developed sepsis on postpartum day 20 and total abdominal hysterectomy was performed. There is also a case report of posterior intrapartum cervicoisthmic uterine rupture in a 42-year-old quadripara without operative procedures and complications in previous pregnancies. 
Cesarean section was performed and a macrosomic male neonate, 4300/54, Apgar score 5/5, was delivered. A hematoma was found in the left parametria, along with posterior cervicoisthmic uterine rupture. The patient underwent total hysterectomy. (7)

In our patient, the risk factors for avulsion and concomitant posterior cervical rupture included prolonged second stage of labor, delivering a macrosomic neonate in a secundipara with deflexion (parietal) cephalic presentation along the distended and thinned posterior uterine wall. Urgent total hysterectomy was performed in dramatic circumstances due to complete unilateral cervical avulsion and tear, with continuous aortal compression, volume replacement and intact coagulation, which certainly contributed to the good final maternal outcome.

\section{References}

1. Ortiz-Mendoza CM, Fernandez Carreno AJ. Uterine avulsion by blunt abdomino-pelvic trauma. Cir Cir 2004;72:499-502.

2. Smith RJ. Avulsion of the nongravid uterus due to pelvic fracture. South Med J 1989;89:70-3.

3. Benijts G, Amy JJ, de Roose J. Traumatic avulsion of the pregnant uterus. Eur J Obstet Gynecol Reprod Biol 1975;5:305-6.

4. Crisp WE, Warrenburg CB, Bowlin B. Intrapartum avulsion of the round ligament. Am J Obstet Gynecol 1962;84:241-3.

5. Fu HC, Ko SF, Ou CY, Hsu TY. Postpartum hemoperitoneum due to avulsion of pelvic uterine adhesion band. South Med J 2006;99:1300-1.

6. Petrović D, Habek D, Vuković Bobić M, Vlahović I. Intrapartal posterior cervicoisthmic uterine rupture : case report. Central Europ J Med 2008;3:374-6.

7. Habek D, Vuković Bobić M, Hrgović Z. Possible feto-maternal clinical risk of the Kristeller's expression. Cent Eur J Med 2008;3:183.-6. 
8. Preschany S, Petzold K, Klostermann K, Stepan H. Intrapartal uterine rupture during vacuum extraction. Arch Gynecol Obstet 2010;282 (Suppl 1):S211.

9. Miller DA, Goodwin TM, Gherman RB, Paul RH. Intrapartum rupture of the nonscarred uterus. Obstet Gynecol 1997;89:671-3.

10. O'Leary JL, O'Leary JA. Manual avulsion of the postpartum uterus, tube and ovary. Obstet Gynecol 1963;22:782-4.

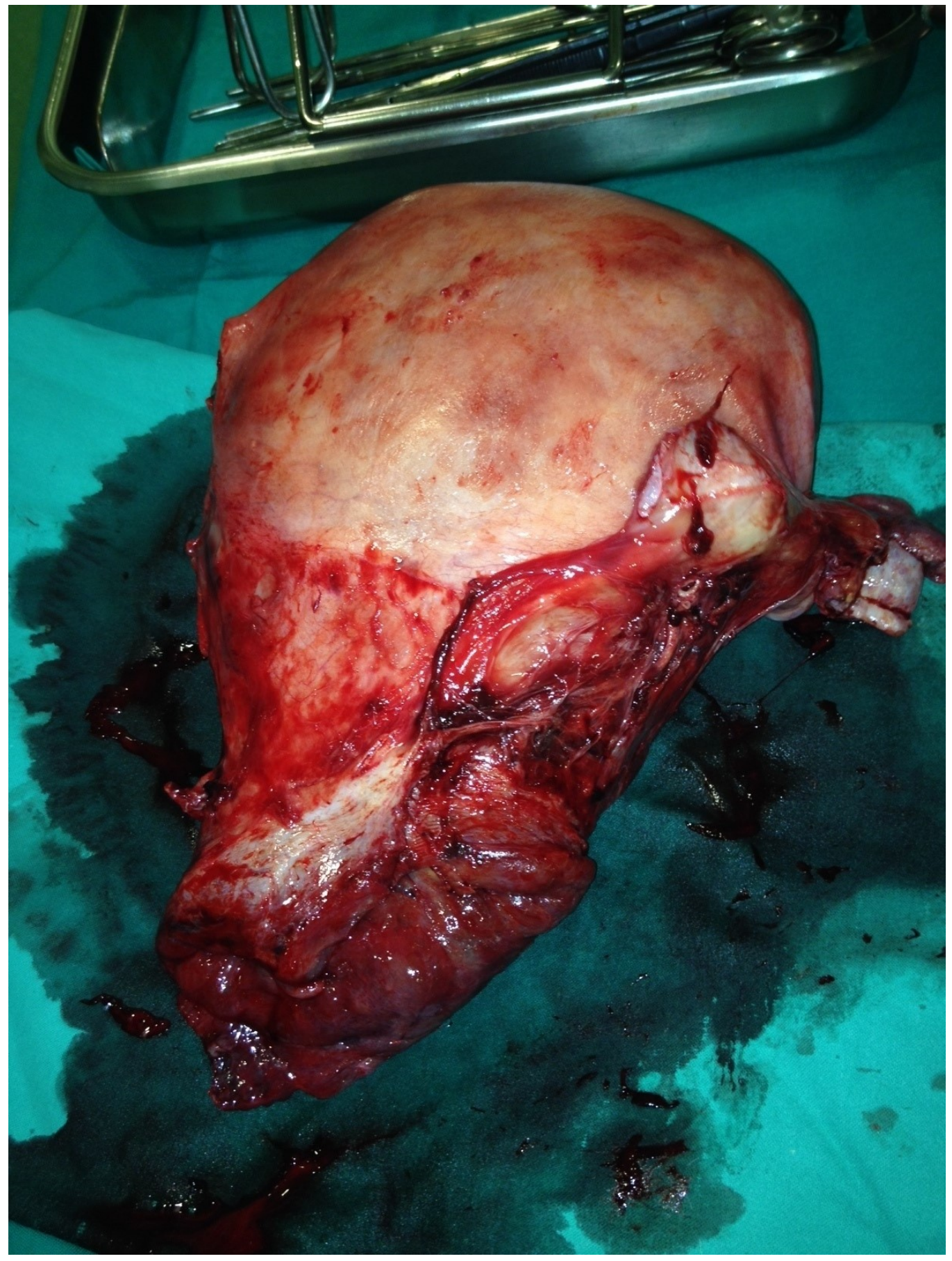


Figure 1. Uterine avulsion with posterior cervical rupture.

Dubravko Habek, Tatjana Pavelić Turudić, Ivan Šklebar

University Department of Obstetrics and Gynecology, Clinical Hospital "Sveti Duh", Zagreb

Correspodence address:

Dubravko Habek

University Department of Obstetrics and Gynecology

Clinical Hospital "Sveti Duh"

Sveti Duh 63, 10000 Zagreb, Croatia

Fax: 0038513745534

Phone: 00385913712112

E-mail: dubravko.habek@os.t-com.hr

Article printed from Signa Vitae: http://www.signavitae.com

URL to article: http://www.signavitae.com/2016/o5/intrapartaluterine-avulsion-with-posterior-cervical-rupture/

Copyright (C) 2015 Signa Vitae. All rights reserved. 